\title{
Neuropsychological and Cognitive Profile of Retired Filipino Boxers
}

\author{
Patricia Ann D. Canto, Alvin Rae F. Cenina, Leah L. Shiong Shu, Michelle M. Anlacan, \\ Lourdes K. Ledesma, Roland Dominic G. Jamora and Carissa Paz C. Dioquino
}

Department of Neurosciences, College of Medicine and Philippine General Hospital, University of the Philippines Manila

\begin{abstract}
Introduction. Boxing is a popular sport in the country. In light of this, risk factors of actual and potential chronic injury among Filipino boxers should be investigated. Repetitive brain trauma from boxing may produce progressive neurological deterioration and is associated with memory disturbances, behavioral and personality changes. This is the first local study that examined and described the cognitive and behavioral profile of retired Filipino boxers where specific variables were measured by making use of several psychological tests.
\end{abstract}

Objectives. This study aimed to describe the neuropsychological and cognitive impairment among retired Filipino boxers and possible risk factors.

Methods. This is a descriptive study on the cognitive and behavioral profile of retired Filipino boxers using standard screening examinations (Neuropsychiatric Index, Symptoms Suggestive of Cognitive Impairment, Hospital Anxiety Depression Scale and Montreal Cognitive Assessment Scale).

Results and Discussion. An increase in times knocked showed a possible relationship with a low MoCA score, with language barrier a factor. SSCl and HADS correlated with the increased rate of being knocked out and with longer years since retirement. Development of cognitive impairment, neuropsychologic and neuropsychiatric features are strongly supported by an increased rate of being knocked out and longer time after retirement, chronic traumatic encephalopathy being a potential consequence of repetitive brain trauma.

Conclusion. An increased rate of being knocked out increases the risk of developing cognitive impairment, neuropsychologic and neuropsychiatric features; with symptoms manifesting years after retirement.

\section{Key Words: chronic brain injury, cognition disorders, boxing}

\footnotetext{
Paper was presented and a finalist at the 34th Philippine Neurological Association Annual Convention, November 2012, Camp John Hay Convention Center, Baguio City, Philippines.

Corresponding author: Patricia Ann D. Canto, MD Department of Neurosciences

Philippine General Hospital

University of the Philippines Manila

Taft Avenue, Ermita, Manila 1000 Philippines

Telefax No.: +632 5548462

Email: trix_canto_08@yahoo.com
}

\section{Introduction}

Boxing is a part of the International Olympic Games and has always intrigued both athletes and audiences despite being an aggressive sport. It is a contact sport and has its risks. In light of its fame, the risk factors of actual and potential injury among Filipino boxers should be investigated. Results will inform and guide clinicians and the general public on health-related issues of boxing.

\section{Chronic Repetitive Brain Injury}

Repetitive brain trauma from boxing may lead to progressive neurological deterioration producing memory disorders, behavioral and personality changes, and gait abnormalities; chronic traumatic encephalopathy (CTE) being associated with these. ${ }^{1}$ Three stages of clinical deterioration in CTE are discussed in a review by Mckee et al. ${ }^{2} \quad$ Affective disturbances and psychotic features demonstrate the first stage. The second stage shows social instability, erratic behavior, memory loss, and initial symptoms of Parkinson disease. The third stage has progressive cognitive dysfunction leading to dementia, fullblown Parkinsonism, speech and gait abnormalities. Furthermore, it reported that personality or behavior changes were seen in $65 \%$ of individuals with neuropathological evidence of CTE, which includes aggression or violence in $70 \%$, confusion in $55 \%$, dysphoria in $48 \%$, paranoia in $42 \%$, irritability in $39 \%$, agitation in $24 \%$, apathy in $9 \%$ and hypersexuality in 3\%.

Forst et $\mathrm{al}^{3}$ in a study using a computer- based neuropsychological testing program (ImPACT) showed that there was no significant difference in immediate recall, working memory or reaction time. However, among 82 boxers, those who were knocked out performed worse in visual- spatial and mathematical exercise two days after, with 18 boxers having impairment in information processing and verbal fluency after a month.

In a research involving amateur boxers with acute traumatic brain injury, test showed that they had significantly different findings in the Categorization Task Test, Digit Symbol Test, Logical Memory: Short Term Memory and Long Term Memory subtests, and Visual Reproduction: Short Term Memory subtest and Long Term Memory subtest. ${ }^{4}$ Heilbronner et $\mathrm{al}^{5}$ noted that the most common neurocognitive deficits in boxers are related to attention, concentration, memory, and motor speed; with 
longer career length showing greater adverse effect. However, the cognitive defects noted was mild compared to control subjects. Moreover, as the disease progresses, symptoms become more severe with broadening of its scope such as worsening of memory, performance of executive tasks, difficulties in language, aggressiveness and irritability, apathy, and disturbance in motor function. ${ }^{1}$

Another review stated that some of the most common presentations of CTE seen in people with repeated traumatic brain injury (TBI) are changes in mood, personality and behavior, ${ }^{6}$ with depression being a common manifestation. Moreover, suicidal tendencies possibly associated with mood change and impulse control deficits are observed frequently. Another article noted that there are numerous neuropsychiatric disturbances associated with TBI, ${ }^{7}$ which are generally observed to be disorders of mood, cognition or behavior, with a classification of neuropsychiatric sequelae of TBI according to phenomenology proposed.

Mood disorders associated with TBI have been reported with the most common being depression and mania. Psychotic symptoms are likewise common and usually manifest as frank delusions, hallucinations and illogical thinking and may be associated with symptoms of agitation, ideas of reference, grimacing, silly giggling, expression of odd ideas, regression and impulsive aggressiveness. Moreover, behavior dyscontrol disorders are likewise present. Both variants may be due to effects of focal or diffuse brain injury resulting in a disruption of neuronal network, creating lapses in cognitive functioning and coarsening of behavior.

The pathologic changes seen in CTE often include cerebral atrophy, cavum septi pellucidi with fenestrations, shrinkage of the mammillary bodies, dense tau immunoreactive inclusions (neurofibrillary tangles, glial tangles, and neuropil neurites), and a TDP-43 proteinopathy. ${ }^{8}$

\section{Boxing and the Brain}

In majority of participants, epidemiologic data studying the occurrence of neurologic injuries in boxing proposed that: 1) irreversible neurologic dysfunction does not occur, 2) the estimated frequency of acute, temporary neurologic injuries in professional boxers is 0.8 brain injuries per 10 rounds and 2.9 brain injuries per 10 boxers, and 3) it is uncommon that permanent neurologic dysfunction occur from brain injury. ${ }^{1}$ In several studies, the weight class and number of rounds, including the number and strength of blows against a boxer was concomitant to the extent of brain damage. It also showed that the heavy weight boxers' blows were stronger than those of lighter weight categories, thereby resulting in a much higher extent of brain damage in the heavy weight division. ${ }^{9}$

The onset of CTE usually begins midlife with gradual progression. A study noted CTE to begin 8 years after retirement (SD 10.7), one-third of which are symptomatic immediately upon retirement, the average duration of which is 20 years among boxers. ${ }^{2}$ The risk of CTE has also been associated with the duration of a boxer's career and with his earlier stamina. In one review article, they cite age $>28$ years, duration of career $>10$ years, number of matches, and poor defensive reflexes as risk factors for developing neuropsychiatric sequel. ${ }^{3}$ Additional factors include frequent knockouts, lengthy sparring sessions, "good staying power," and carrying the apolipoprotein E4 genotype (single or double).

There is limited epidemiological data on the prevalence of CTE among amateur and professional boxers. In the 1930s - 1950s, $17 \%$ of professional boxers in Britain had clinical evidence of CTE. ${ }^{10}$ In another study 10 to $20 \%$ of professional boxers suffer from persistent neuropsychiatric sequelae, which included motor, cognitive, and behavioral changes. ${ }^{11}$

Boxing has developed rules to ensure the safety of the athletes as evidenced by stricter rules in amateur bouts. These protective measures include compulsory wearing of a head guard, more heavily cushioned gloves, and setting shorter and fewer rounds. Bouts consist of three rounds of three minutes in the Olympic and Commonwealth Games, and three rounds of three minutes in a national ABA (Amateur Boxing Association) bout, each with a one-minute interval between rounds.

In contrast, bouts are usually much longer in professional boxing, typically ranging from ten to twelve rounds, lasting 3 minutes each. The use of headgears is also not allowed in professional boxing. In amateur boxing, the following measures are also allowed: stopping the bout in accordance with the "outclassed rule", the option for a boxer to interrupt the bout himself and, the option for the ringside doctor to intervene.

This study will examine and describe the cognitive and behavioral profile of retired Filipino professional boxers using standard neurologic examination, Neuropsychiatric Inventory (NPI), Symptoms Suggestive of Cognitive Impairment (SSCI), Montreal Cognitive Assessment Scale (MoCA), and Hospital Anxiety and Depression Scale (HADS). It also aims to describe its possible risk factors including age, professional boxing, duration of career, weight division, number of fights, and number of knockouts endured. This is the first local study that examined and described the cognitive and behavioral profile of retired Filipino boxers where specific variables were measured by making use of several psychological tests.

\section{Methods}

\section{Methodology}

This is a descriptive study on the cognitive and neuropsychological profile of retired Filipino boxers using standard screening examinations such as the NPI, SSCI, HADS and MOCA scales. 


\section{Sample Selection}

A list of amateur and professional boxers were obtained from the Amateur Boxer's Association of the Philippines (ABAP), the Philippine Sports Commission (PSC) and the Games and Amusements Board (GAB). Study participants were recruited from four ELORDE gyms and consent for inclusion into the study was secured. A total of nineteen (19) professional boxers who are current trainers at the different ELORDE gyms were included in the study. Participants were examined at their respective gyms during their free periods and were screened for cognitive and neuropsychological functioning. Signed consent was taken.

\section{Population}

\section{Inclusion criteria:}

Retired boxers, both professional and amateur, who understands both English and Filipino.

\section{Exclusion criteria:}

Known co-morbid cerebrovascular disease or other documented CNS diseases such as CNS tumors, CNS infection, Demyelinating Diseases, Stroke, vehicular accidents, syphilis, HIV-AIDS.

\section{Screening Tools}

Baseline demographic data and possible risk factors were obtained including a questionnaire on cognitive and neuropsychological symptoms, which were answered by the patient and his watcher. Standard physical and neurologic examinations were performed, followed by the administration of the NPI, SSCI, HADS and MoCA.

\section{NPI. (Neuropsychiatric Inventory).}

The NPI assessed 12 types of neuropsychiatric disturbances including delusions, hallucinations, agitation/aggression,

irritability/lability, aberrant motor activity, anxiety, euphoria, apathy, disinhibition, nighttime behavior disturbances and appetite/eating abnormalities. The caregiver rated the frequency and severity of each abnormality, as well as distress associated with each behavioral symptom. The total score for each domain was calculated by multiplying the frequency by the severity, and the total score is the sum of the scores of all the domains.

\section{SSCI. (Symptoms Suggestive of Cognitive Impairment).}

This is a caregiver or self-report interview questionnaire meant to elicit observed changes in cognition, behavior and the performance of activities of daily living. This was used to qualitatively evaluate the participant's cognitive function at home and the community.

\section{MoCA. (Montreal Cognitive Assessment scale).}

This is a brief assessment of general intellectual function including orientation, immediate and delayed recall, attention and calculation, and different components of language. A score of less than 26 is considered abnormal. The English version of the MoCA will be used in this study since no validated Filipino version exists.

\section{HADS. (Hospital Anxiety and Depression Scale.)}

This is a 14 item screening tool for detecting states of depression and anxiety. Initially developed in 1983, it has been found to be valid for use in both psychiatric and nonpsychiatric outpatient clinics, and even in the community setting. A validated Filipino version was used. The maximum score for anxiety and depression is 21 each. A score of 11 to 21 is considered abnormal, with scores of 8 to 10 as borderline abnormal. In this study, the patient would be considered to have depressive symptoms if the score of HADS-D is more than seven.

\section{Results}

\section{Demographics}

Sample demographics including age onset of boxing, retirement age, sparring hours per week, boxing years \& fights (amateur and professional), and trainer years are detailed in Table 1.

Table 1. Demographics

\begin{tabular}{lc}
\hline \multicolumn{1}{c}{ BOXING DATA } & AVERAGE (RANGE) \\
\hline Age of Onset of Boxing & $16(10-23)$ \\
Age at Retirement & $27(22-36)$ \\
Sparring hours per week & $4.3(\mathbf{1 - 1 0 . 5 )}$ \\
Total years as boxer & $10.4(2-31)$ \\
Years as Amateur Boxer & $1.5(0-5)$ \\
Years as Professional Boxer & $9(2-29)$ \\
Total Amateur Fights & \\
Wins & $15(0-100)$ \\
Draws & $0.47(0-4)$ \\
Losses & $4.7(0-27)$ \\
Times Knocked Out & $0.64(0-9)$ \\
Years since retirement & $11.7(1-32)$ \\
Total Professional Fights & \\
Wins & $22(0-70)$ \\
Draws & $2.04(0-7)$ \\
Losses & $11.3(0-50)$ \\
Times Knocked Out & $1.38(0-5)$ \\
Total years as boxing trainer & $8.05(0-32)$ \\
\hline
\end{tabular}

\section{Cognitive}

\section{Montreal Cognitive Assessment}

The Montreal Cognitive Assessment (MoCA) was used to evaluate the intellectual function of the boxers included in the study (Table 2). Each boxer was screened and given ample time to answer. The average MoCA score of the boxers was 19.6. Only one boxer was within normal range (26), while the rest had scores below cut-off (the only college graduate in the group). 
Table 2. MoCA Score and educational attainment

\begin{tabular}{lc}
\hline EDUCATIONAL ATTAINMENT & MoCA SCORE RANGE \\
\hline Overall (19) & $11-26$ \\
Elementary Undergraduate/Graduate (8) & $13-22$ \\
High school Undergraduate/Graduate (7) & $20-24$ \\
College Undergraduate/Graduate (4) & $11-26$ \\
\hline
\end{tabular}

Among those who were elementary undergraduates (EU)/elementary graduates (EG), the average MoCA score was 15.5, whereas those of high school undergraduates $(\mathrm{HU}) /$ high school graduates (HG) and college undergraduates (CU)/college graduates (CG), were 23 and 20, respectively. Among all boxers, the average length of boxing and trainer career was 20.3 years whereas that of active boxing career (amateur and professional) was 11.5. In this group the shortest (3 years) and longest (37 years) boxing careers both showed the same MoCA score (13). The highest MoCA average was among the high school undergraduate/ graduate group (22.7) and the lowest score among the elementary undergraduate/ graduate group (15.5). Age at onset of boxing was earliest among the college undergraduate/ graduate group (12.2 years old). Total years of boxing are almost the same throughout all groups. The longest career as an amateur boxer was among the college undergraduate/ graduate group (3.2 years) while longest career as a professional was among the high school undergraduate/ graduate group (9.7 years). The highest number of years since retirement was among the high school undergraduate/ graduate group (16.6), and the lowest was among the elementary undergraduate/ graduate group (7). The highest number of total fight was seen in the high school undergraduate/ graduate group (77.4), with the number of amateur fights highest in the college undergraduate/ graduate group (43) and professional fights highest in the high school undergraduate/ graduate group (56.4). Times knocked out were lowest among the high school undergraduate/ graduate group (1.4) and highest among the elementary undergraduate/ graduate group (2.5).

\section{Symptoms Suggestive of Cognitive Impairment}

The Symptoms Suggestive of Cognitive Impairment (SSCI) test was used to evaluate the boxers' cognitive function at home and in the community. Among all the boxers screened, 11 had subjective complaints in the neuropsychological domain, relating to memory impairment interfering with work and social functioning, misplacing of important items and language problems such as word finding difficulties and substitution of inappropriate words. Four of these boxers also had subjective complaints in the neuropsychiatric domain, relating to depression, irritability or lability and changes in sleep patterns. None of them however had any problems in the activities of daily living domain.
A total of 11 boxers presented with neuropsychological features, as demonstrated in Table 3. In this group of boxers, the average years since retirement and times knocked out were higher among those with neuropsychological features. Among those listed above, four of the boxers also presented with neuropsychiatric features, which is shown in Table 4. The features presented were depression, irritability; changes in sleep pattern, and anxiety. The average numbers of years since retirement and the number of times knocked out are higher among those with neuropsychiatric features compared to those without the features.

Table 3. SSCI: Boxers with and without neuropsychological features

\begin{tabular}{lll}
\hline \multicolumn{1}{c}{ BOXING } & $\begin{array}{c}\text { AVERAGE } \\
\text { (RANGE) }\end{array}$ & $\begin{array}{c}\text { AVERAGE } \\
\text { (RANGE) } \\
\text { No }\end{array}$ \\
DEMOGRAPHICS/MoCA & $\begin{array}{c}\text { Neuropsychological } \\
\text { Features }\end{array}$ & $\begin{array}{c}\text { Neuropsychological } \\
\text { Features }\end{array}$ \\
\hline MoCA Score & $19.4(13-23)$ & $19.9(11-26)$ \\
Age of onset of boxing & $15.5(10-22)$ & $15.3(12-20)$ \\
Total years of boxing & $9.8(3-24)$ & $13.9(7-31)$ \\
Amateur & $1.3(0-5)$ & $2.2(0-4)$ \\
Professional & $7.5(2-20)$ & $11.8(5-29)$ \\
Years since retirement & $13.4(3-27)$ & $9.0(1-32)$ \\
Total boxing and trainer years & $18.1(3-36)$ & $21.9(7.5-39)$ \\
Sparring hours per week & $3.1(1-6)$ & $4.4(1-4.5)$ \\
Total fights & $43.4(8-119)$ & $78.5(8-180)$ \\
$\quad$ Amateur & $16.9(0-110)$ & $23.8(0-80)$ \\
Professional & $26.5(7-71)$ & $54.4(7-122)$ \\
Times knocked out & $2.6(0-11)$ & $1.0(0-2)$ \\
\hline
\end{tabular}

Table 4. SSCI: Boxers with both neuropsychological and neuropsychiatric features versus those with neuropsychological features only

\begin{tabular}{|c|c|c|}
\hline $\begin{array}{c}\text { BOXING } \\
\text { DEMOGRAPHICS/MoCA }\end{array}$ & $\begin{array}{c}\text { AVERAGE } \\
\text { (RANGE) } \\
\text { Both } \\
\text { Neuropsychological } \\
\text { and Neuropsychiatric } \\
\text { Features }\end{array}$ & $\begin{array}{l}\text { Neuropsychological } \\
\text { Features Only }\end{array}$ \\
\hline MoCA Score & $20.25(17-22)$ & $19.5(11-26)$ \\
\hline Age of onset of boxing & $15.25(10-20)$ & $15.5(10-20)$ \\
\hline Total years of boxing & $10.75(5-16)$ & $11.4(3-31)$ \\
\hline Amateur & $0.75(0-3)$ & $2.0(0-5)$ \\
\hline Professional & $10(5-13)$ & $9.8(2-29)$ \\
\hline Years since retirement & $16.5(7-24)$ & $10.9(1-32)$ \\
\hline Total boxing and trainer years & $23.0(16-36)$ & $18.8(3-39)$ \\
\hline Sparring hours per week & $2.0(1-3)$ & $4.1(1-9)$ \\
\hline Total fights & $38.5(12-95)$ & $63.7(15-180)$ \\
\hline Amateur & $10.7(0-43)$ & $22.3(0-110)$ \\
\hline Professional & $27.7(12-71)$ & $41.1(7-122)$ \\
\hline Times knocked out & $4.2(0-11)$ & $1.6(0-4)$ \\
\hline
\end{tabular}

\section{Behavioral}

\section{Hospital Anxiety and Depression Scale}

The Hospital Anxiety and Depression Scale (HADS) was used to screen for depression and anxiety among the participants. Among the screened boxers, one had a HADS- 
D score of eight (8) and one had a HADS-A score of eleven (11), which were both considered abnormal in this study. The rest of the boxers had a HADS-D and HADS-A score ranging from 0-5 and 0-7, respectively.

The boxer with the abnormal HADS-D score had a MoCA score of twenty-six (26), which is the highest among all the boxers. He had no neuropsychological or neuropsychiatric complaints based on SSCI (Table 5). On the other hand, the boxer with the abnormal HADS-A score had a MoCA score of twenty (20). Moreover, he had neuropsychological complaints related to memory impairment and misplacing items as well as neuropsychiatric complaints related to depression and anxiety, based on the SSCI (Table 6).

Table 5. HADS-D: Demographics of boxer with abnormal HADS-D

\begin{tabular}{ll}
\hline BOXING DEMOGRAPHICS & \\
\hline Age of onset of boxing & 12 years old \\
Total years of boxing & 11 years \\
$\quad$ Amateur & 4 years \\
Professional & 7 years \\
Years since retirement & 1 year \\
Total boxing and trainer years & 12 years \\
Sparring hours per week & 4.5 hours \\
Total fights & 43 fights \\
Amateur & 29 fights \\
Professional & 14 fights \\
\hline
\end{tabular}

Table 6. HADS-A: Demographics of boxer with abnormal HADS-A

\begin{tabular}{ll}
\hline BOXING DEMOGRAPHICS & \\
\hline Age of onset of boxing & 14 years old \\
Total years of boxing & 16 years \\
$\quad$ Amateur & 3 years \\
Professional & 13 years \\
Years since retirement & 11 years \\
Total boxing and trainer years & 19 years \\
Sparring hours per week & 3 hours \\
Total fights & 92 fights \\
$\quad$ Amateur & 40 fights \\
Professional & 14 fights \\
\hline
\end{tabular}

\section{Neuropsychiatric Inventory}

Among nineteen (19) boxers, only one (1) boxer presented with abnormal behavior (irritability/ lability) (Table 7).

This boxer had a MoCA score of 17, age onset of boxing at 20, 10 years boxing career ( 0 amateur, 10 professional), 19 years since retirement, 16 total years as boxer and trainer, 1 hour sparring per week, and 35 total fights (0 amateur, 35 professional). This boxer also presented with neuropsychological features (language problems), as well as neuropsychiatric features (irritability).
Table 7. NPI: Irritability/lability scoring of boxer with abnormal NPI

\begin{tabular}{ll}
\hline Frequency & 2 \\
Severity & 1 \\
Total & 2 \\
Caregiver Distress & 2 \\
\hline
\end{tabular}

\section{Discussion}

The Montreal Cognitive Assessment (MoCA) was designed as a rapid screening for mild cognitive dysfunction, which focused on various cognitive domains. Among nineteen boxers, only one person got a score of 26, denoting mild cognitive dysfunction. Of note is the fact that the sole boxer who had a normal MoCA score was a college graduate, with no other boxer graduating with a degree. Another striking data was that the highest average MoCA score was among the high school undergraduate/ graduate group. This may be attributed to the small sample size of the college undergraduate/ graduate group, and that the groups are not entirely comparable due to this.

Length of boxing career demonstrated no relationship with the MoCA score. Sparring hours per week was lowest among the elementary undergraduate/ graduate group, and highest among the college undergraduate/ graduate group. The number of total fights was directly proportional to the MoCA scores, with the highest scores among the high school undergraduate/ graduate group and the lowest among the elementary undergraduate/ graduate group.

Another relationship of note is that the group with the lowest MoCA score had the highest average times knocked out. This could likely point to times knocked out having an effect on the MoCA scores. Cognitive impairment may therefore be linked to the number of times knocked out.

MoCA item analysis showed that all boxers had low scores in both storage/retrieval and language with no relation to their educational status. This may be explained by the fact that the questionnaire given to the boxers was in English (there being no validated Filipino version of the questionnaire as of the moment). This gap in language could be the main reason why all but one of the boxers demonstrated mild cognitive dysfunction. Another factor may be the small sample size, which may not be representative of the population.

The Symptoms Suggestive of Cognitive Impairment (SSCI) test evaluated for neuropsychological and neuropsychiatric features, as well as hampering in activities of daily living. Among all boxers, eleven (11) were reported to have neuropsychological features. All demographics, except for the number of times knocked out showed no difference from the average of all boxers. However, the number of time knocked out among all those with neuropsychological features was higher compared to the rest of the boxers. This could mean that the risk of developing neuropsychological features in a boxer is increased by the 
number of times he was knocked unconscious. Moreover, the number of years since retirement was slightly higher among those with both neuropsychological features as compared to the general average. This may indicate that neuropsychological features may be more evident over time.

Among all boxers, four were reported to have neuropsychiatric features such as depression, irritability, changes in sleep pattern, and anxiety. Furthermore, this group presented with slightly longer boxer/ training years compared to all boxers, The average number of times knocked out was more than twice the general average. This may again indicate that the number of times being knocked unconscious increases the risk of developing neuropsychiatric features. Similarly, these boxers with neuropsychiatric features were retired for a longer time compared to those without, thus, further supporting symptom manifestation over time.

Being punched in the head with secondary loss of consciousness may be a risk factor for developing neuropsychological and neuropsychiatric features as shown by its direct relationship with times knocked out. In addition, these features are more obvious over time as demonstrated by those boxers who were retired longer.

The Hospital Anxiety and Depression Scale (HADS) showed only one boxer with depression, and only one boxer with anxiety. The demographics of the boxer with depression mainly fall within the general average, and only had two episodes of being knocked out. This boxer had the highest MoCA score and was the one person who was a college graduate and did not have any symptoms based on SSCI. This result shows no apparent relationship. Furthermore, upon his inclusion in this study, this boxer was only retired for one year and thus, may not have manifested with any possible neuropsychological and neuropsychiatric symptoms. The boxer who had anxiety also had his demographics within the general average, except for the increased number of times knocked out. This boxer presented with neuropsychological and neuropsychiatric features. This result could be related to his neuropsychological and neuropsychiatric symptoms.

The Neuropsychiatric Inventory Questionnaire showed only one boxer with abnormal behavior in the irritability/ lability domain. Interestingly, this boxer also presented with both neuropsychological and neuropsychiatric features. Though he had no episodes of being knocked out, he was retired for 19 years, which is higher compared to the average years of retirement of all boxers. This could explain why this boxer already manifested with neuropsychological and neuropsychiatric symptoms and tested positive on the irritability domain of the NPI.

It is an established fact that repeated head trauma increases an individual's risk of CTE. This has been shown in athletes who participate in contact sports such as American football, boxing, hockey, soccer and professional wrestling. Several other factors such as number of times knocked out, age started, length of career, sparring duration and number of fights competed may also influence the individual's risk for CTE. Traumatic injury may start a cascade of injury during brain development, and may be compounded through the years. ${ }^{6}$ Conversely however, at younger ages, the brain has more plasticity, with the ability to manage injury than as compared to the adult brain. The length of career is another risk factor, predisposing to prolonged exposure, which may thereby cause more severe CTE. ${ }^{12}$ In another study of 39 boxers, it was noted that they had an average career of 14.4 years with a range of 4-25 years and that they started between 11 and 19 years. $^{2}$

In one study, ${ }^{13}$ there were no significant effects found when examining the relationship between number of knockouts, number of wins and losses, number of bouts where the referee stopped the contest and the weight at which the boxer competes and the neuropsychological functioning. This study however was conducted only among amateur. In another review, it was noted that there seems to be an association between the incidence of abnormality, length of career, number of bouts and number of knockouts, and that it appears to follow the model of cumulative effect wherein blows to the head have an additive harmful effect. ${ }^{14}$

\section{Conclusion}

The Montreal Cognitive Assessment scores showed a relationship with their increased episodes of being knocked out. This may represent cognitive impairment secondary to repetitive knockouts. However, all but one of the boxers had below normal MoCA scores, signifying mild cognitive impairment. Language barrier (the exam being in English) could have had a possible effect thereby most of the boxers getting low scores.

Symptoms Suggestive of Cognitive Impairment showed eleven boxers with neuropsychologic features and four with neuropsychiatric feature with knockouts of 2.6 and 4.2, respectively, which is more than the general average. This shows that being knocked unconscious may increase the risk of developing neuropsychological and neuropsychiatric symptoms. This is further reinforced by the HADS-A result of one boxer with anxiety that had eleven episodes of being knocked out. Furthermore, the average years since retirement is higher in these two groups compared to the rest of the boxers indicating that manifestation of neuropsychologic features and neuropsychiatric features appear at a later time. The development of CTE, on average, develops 17.5 years after retirement. ${ }^{6}$ This average is quite close to the average of the latter group, which further supports that a boxer develops the symptoms years after retirement.

An increased rate of being knocked out therefore increases the risk of developing cognitive impairment, neuropsychologic and neuropsychiatric features. This is 
further supported by a study wherein, despite the presence of headgear, traumatic brain injury was still detected among amateur boxers who were punched in the head and presented with patterns of impaired performance in planning, attention, and memory capacity when compared with the control group. ${ }^{15}$ Another study reiterates that chronic traumatic encephalopathy is a potential consequence of repetitive brain trauma. ${ }^{16}$

\section{Recommendations}

A validated Filipino version of the MoCA test should be used for better cognitive evaluation of boxers. Language barrier presents a very valid argument, and misrepresentation as having mild cognitive impairment is a deterrent for its further use locally.

A prospective study of boxers is recommended since several authors noted that chronic traumatic encephalopathy sets in 17.5 years after retirement. This would give a better understanding of its progression and the clinical course of symptom development may be better represented.

\section{References}

1. Stern RA, Riley DO, Daneshvar DH, Nowinski CJ, Cantu RC, McKee AC. Long-term consequences of repetitive brain trauma: chronic traumatic encephalopathy. PMR. 2011; 3(10Suppl 2):S460-7.

2. McKee AC, Cantu RC, Nowinski CJ, et al. Chronic traumatic encephalopathy in athletes: progressive tauopathy following repetitive head injury. J Neuropathol Exp Neurol. 2009; 68(7):709-35.

3. Förstl H, Haass C, Hemmer B, Meyer B, Halle M. Boxing - acute complications and late sequelae - from concussion to dementia. Dtsch Arztebl Int. 2010; 107(47):835-9.

4. Matser EJ, Kessels AG, Lezak MD, Troost J, Jordan BD. Acute traumatic brain injury in amateur boxing. Phys Sportsmed. 2000; 28(1):87-92.

5. Heilbronner RL, Bush SS, Ravdin LD, et al. Neuropsychological consequences of boxing and recommendations to improve safety: a National Academy of Neuropsychology education paper. Arch Clin Neuropsychol. 2009; 24(1):11-9.

6. Gavett BE, Stern RA, McKee AC. Chronic traumatic encephalopathy: a potential late effect of sport-related concussive and subconcussive head trauma. Clin Sports Med. 2011; 30(1):179-88.

7. Rao V, Lyketsos C. Neuropsychiatric sequelae of traumatic brain injury. Psychosomatics. 2000; 41(2):95-103.

8. Gavett BE, Cantu RC, Shenton M, et al. Clinical appraisal of chronic traumatic encephalopathy: current perspectives and future directions. Curr Opin Neurol. 2011; 24(6):525-31.

9. Unterharnscheidt F. A neurologist's reflections on boxing. Rev Neurol. 1995; 23(123):1027-32.

10. McCrory P Zazryn T, Cameron P. The evidence for chronic traumatic encephalopathy in boxing. Sports Med. 2007; 37(6):467-76.

11. Rabadi MH, Jordan BD. The cumulative effect of repetitive concussion in sports. Clin J Sport Med. 2001; 11(3):194-8.

12. Saulle M, Greenwald BD. Chronic Traumatic Encephalopathy: A Review. Rehabil Res Pract. 2012; 2012:816069.

13. Brooks N, Kupshik G, Wilson L, Galbraith S, Ward R. A neuropsychological study of active amateur boxers. J Neurol Neurosurg Psychiatry. 1987; 50(8):997-1000.

14. Butler RJ. Neuropsychological investigation of amateur boxers. Br J Sports Med. 1994; 28(3):187-90.

15. Matser EJ, Kessels AG, Lezak MD, Troost J, Jordan BD. Acute traumatic brain injury in amateur boxing. Phys Sportsmed. 2000; 28(1):87-92.

16. Lakhan SE, Kirchgessner A. Chronic traumatic encephalopathy: the dangers of getting "dinged". Springerplus. 2012; 1:2. 